\title{
COMPENSATION OF THE IONOSPHERIC EFFECTS ON SAR INTERFEROGRAM BASED ON RANGE SPLIT-SPECTRUM AND AZIMUTH OFFSET METHODS- A CASE STUDY OF YUSHU EARTHQUAKE
}

\author{
Y. F. He ${ }^{1}$, W. Zhu ${ }^{1, *}$, Q. Zhang ${ }^{1}$, W. T. Zhang ${ }^{1}$ \\ ${ }^{1}$ School of Geology Engineering and Geomatics, Chang'an University, Xi'an, China, 710054, zhuwu@ @chd.edu.cn, \\ hyf_chd@163.com, zhangqinle@263.net.cn
}

Commission III, WG III/3

KEYWORDS: InSAR, Ionospheric effects, Range split-spectrum, Azimuth offset, Ionosphere correction, Yushu earthquake.

\begin{abstract}
:
InSAR technique can measure the surface deformation with the accuracy of centimeter-level or even millimeter and therefore has been widely used in the deformation monitoring associated with earthquakes, volcanoes, and other geologic process. However, ionospheric irregularities can lead to the wavy fringes in the low frequency SAR interferograms, which disturb the actual information of geophysical processes and thus put severe limitations on ground deformations measurements. In this paper, an application of two common methods, the range split-spectrum and azimuth offset methods are exploited to estimate the contributions of the ionosphere, with the aim to correct ionospheric effects in interferograms. Based on the theoretical analysis and experiment, a performance analysis is conducted to evaluate the efficiency of these two methods. The result indicates that both methods can mitigate the ionospheric effect in SAR interferograms and the range split-spectrum method is more precise than the other one. However, it is also found that the range split-spectrum is easily contaminated by the noise, and the achievable accuracy of the azimuth offset method is limited by the ambiguous integral constant, especially with the strong azimuth variations induced by the ionosphere disturbance.
\end{abstract}

\section{INTRODUCTION}

In the low frequency SAR differential interferometry, spatial variations of the electron density level for the ionosphere cause the group delay, phase advance and faraday rotation during the satellite signal propagation, resulting in the generation of wavy fringes in the InSAR interferograms and even masking the surface deformation information, which greatly affects the analysis of earthquake deformation, volcanic movement and other surface movement analysis (Rosen, 2010).

In recent years, several possible methods for the InSAR ionospheric correction have been published, which contains the autofocus techniques to estimate the absolute ionosphere and other techniques to estimate the differential one (Meyer, 2010). However, the former techniques are not able to capture the relatively small irregularities with sufficient accuracy. The latter are the most common techniques to compensate the ionospheric blurring, which contains the group delay, the Faraday rotation, azimuth offset method, the range split-spectral methods and so on (Gomba, 2017). The group delay method requires establishing a linear regression between the group delay and the phase advance to estimate the ionosphere phase delay. In fact, the unwanted accuracy of this method is derived for the low precision of the group delay and the low spatial resolution (Brcic, 2010). The Faraday rotation method exploits the quad-polarized measurements to estimate the Faraday rotation angle and the TEC level. However, the Global application of this method is limited due to the weak magnetic field near the equator (Wright, 2003). The azimuth offset method employs the approximate linear relationship between the ionosphere-induced azimuth offset and the ionospheric phase delay to suppress the ionospheric contribution (Jung, 2013). With the dispersive nature of radar signals, the ionospheric phase delay in the microwave signal is inversely proportional to the signal frequency, which can be exploited to estimate the ionospheric phase delay (Bamler, 2005). Although quite a few techniques are exploited to correct the ionospheric irregularities, an evaluation of their performances and suitability for an operational environment is still missing.

In this paper, we exploit the most common methods which are the range split-spectral method and the azimuthal offset method to estimate the differential ionospheric path delay with the various ground and ionospheric blurring exiting together. Based on the theoretical analysis and an experiment of L-band ALOS PALSAR images, a performance analysis is conducted to evaluate the efficiency of these two methods. The structure of this paper is arranged as follow: firstly, the experimental methods, including range split-spectrum and azimuth offset, are described; then, the experiment is shown. In the end, we analyzed and discussed the result of the experiment.

\section{METHODOLOGY}

\subsection{The Range Split-spectrum Method}

Based on the dispersive nature of the ionosphere for radar signals, SAR interferometric phase formed from two SAR acquisitions can be divided into the dispersive phase equals the ionospheric delay phase and non-dispersive phase including the topography, deformation phase and topographic path delay. Two components with different frequency behavior are exploited by the range split-spectrum to separate the ionospheric delay phase from other phases. At first, two non-overlapping sub-bands with slightly different carrier frequencies are generated through a band-pass filter. The phases of sub-band interferograms are as followings (Gomba, 2016). 


$$
\begin{gathered}
\Delta \varphi_{1}=\Delta \varphi_{\text {non-disp }} \frac{f_{1}}{f_{0}}+\Delta \varphi_{\text {iono }} \frac{f_{0}}{f_{1}} \\
\Delta \varphi_{2}=\Delta \varphi_{\text {non-disp }} \frac{f_{2}}{f_{0}}+\Delta \varphi_{\text {iono }} \frac{f_{0}}{f_{2}}
\end{gathered}
$$

Where $f_{1}=$ center carrier frequency of sub-band SLC1s

$f_{2}=$ center carrier frequency of sub-band SLC2s

$f_{0}=$ center carrier frequency of full-band SLCs

$\Delta \varphi_{1}=$ the phase of interferogram corresponding to sub-band with center frequencies of $f_{1}$

$\Delta \varphi_{2}=$ the phase of interferogram corresponding to sub-band with center frequencies of $f_{2}$

Absolutely, we can capture the differential dispersive component and nondispersive component of delay by a simply mathematical inverting of the above two equations.

$$
\begin{aligned}
& \Delta \varphi_{\text {iono }}=\frac{f_{1} f_{2}}{f_{0}\left(f_{1}{ }^{2}-f_{2}{ }^{2}\right)}\left(\Delta \varphi_{1} f_{2}-\Delta \varphi_{2} f_{1}\right) \\
& \Delta \varphi_{\text {non-disp }}=\frac{f_{0}}{\left(f_{1}{ }^{2}-f_{2}{ }^{2}\right)}\left(\Delta \varphi_{2} f_{2}-\Delta \varphi_{1} f_{1}\right)
\end{aligned}
$$

Where $\Delta \varphi_{\text {iono }}=$ the differential dispersive component

$$
\Delta \varphi_{\text {non-disp }}=\text { the non-dispersive component of delay }
$$

Although the mathematical theory is quite easily understood, one careful operation is quite necessary to be done in the practical implementation. The schematic representation to estimate the differential ionospheric phase delay on SAR interferometry by using the range split-spectrum method is shown in Figure 1. The practical operation mainly includes three aspects. Firstly, we split the range-spectrum of SLCs to generate the sub-band SLCs and resample them using the offsets from the co-registration of the full-band images; secondly, sub-band unwrapped interferograms using DEM data are used to separate the ionospheric delay phase from other phases through the mathematical transformation. The ionospheric delay phases acquired initially are too noisy to ignore more fine operations to smooth and the phase unwrapping errors should be removed. In the end, the ionosphere-contaminated interferogram is corrected through subtracting the separated ionospheric delay.

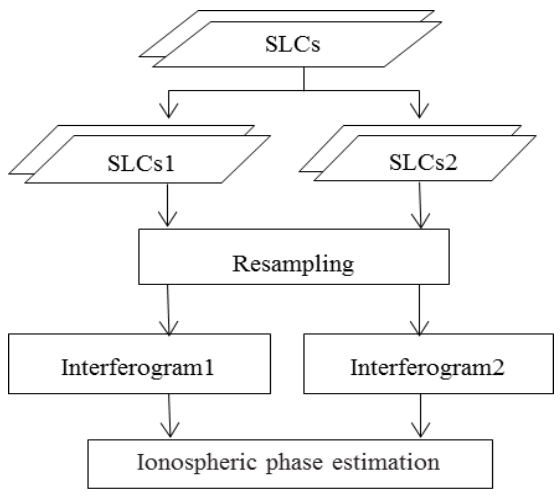

Figure 1. Overview of IPS processing flowchart

The achievable accuracy of this method is derived for some operations as followings. The proper bandwidth and the center carrier frequency are quite important to suppress noise amplification and maintain image information. Meanwhile, in order to improve the result, the interferometric coregistration is carefully analyzed to estimate accurate offsets to recover the coherence (Fattahi, 2017). If the coherence of interferograms formed by sub-bands SLCs are not high enough, an adaptive filter would be required to suppress the noise. Of course, the ionospheric phase delay may be confusing without the step for the correction of phase unwrapping errors. The high accuracy of this method performed here is based on the above operations precisely completed.

The range split-spectrum can correct the error induced by ionosphere at different bandwidth of images theoretically, but the narrower bandwidth requires higher coherence due to the larger noisy amplification in the practical operation. However, interferograms are not absolute phases, and thus the differential dispersive component can be only known to within a constant offset.

\subsection{The Azimuth Offset Method}

The research indicates that there is an approximate linear relationship between the ionosphere-induced azimuth offset and the ionospheric phase delay. Thus, it is capable to use the prominent azimuth offset induced by the ionospheric disturbance on SAR interferograms to suppress the effect of ionosphere. This linear relationship can be constructed just as:

$$
\Delta \varphi_{\text {iono }}=\propto \int \Delta x_{\text {offset }} d x+c
$$

Where $\Delta x_{\text {offset }}=$ the interferometric azimuth offset $x=$ the azimuth direction.

$\alpha=$ the system- and geometry-dependent scaling factor $c=$ the integration constant

After estimating these two parameters, the ionospheric phase delay is inverted by InSAR azimuth offset, and then is used to mitigate the ionospheric effects of InSAR. The designed flowchart of Interferometric phase estimation is shown in Figure 2. For the azimuth offset method, it is important that the azimuthal offset, scale factor and accuracy of integral constant can directly affect the performance of azimuthal offset method.

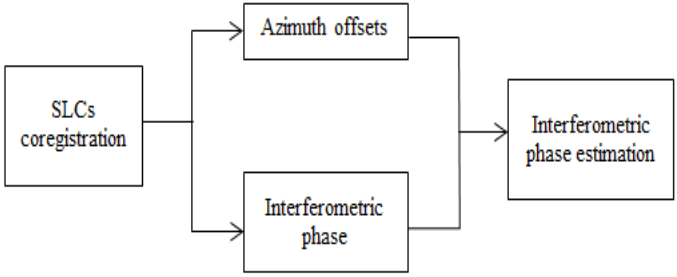

Figure 2. Overview of IPS processing flowchart

\section{EXPERIMENT AND ANALYSIS}

\subsection{Data Collection}

On April 14, 2010, an earthquake struck the Yushu in northwest of China. Our study applied L-band ALOS / PALSAR data acquired from the ascending path 487 before and after the earthquake, and the designed interferometric parameters are shown in Table 1.

\begin{tabular}{lllll}
\hline No & Master & Slave & $\begin{array}{l}\mathbf{B}_{\mathbf{T}} \\
\text { (days) }\end{array}$ & $\begin{array}{l}\mathbf{B}_{\mathbf{P}} \\
(\mathbf{m})\end{array}$ \\
\hline 1 & $15 / 01 / 2010$ & $17 / 04 / 2010$ & 92 & 671 \\
\hline 2 & $15 / 01 / 2010$ & $18 / 07 / 2010$ & 184 & 865 \\
\hline
\end{tabular}

Table 1. Interferometric parameters for Yushu earthquake area. 
In Table $1, \mathrm{~B}_{\mathrm{T}}$ stands for the temporal baseline and $\mathrm{B}_{\mathrm{P}}$ stands for perpendicular baseline. Interferometric pair 1 is disturbed by the ionospheric irregularities but pair 2 is not, so the former pair can be used to evaluate the performance of ionospheric correction for pair 2 by using two methods. Images of pair 2 acquired in two modes, fine beam single polarization (FBS) and fine beam dual polarization (FBD) with different bandwidth.

Figure 3.a displays the ground coverage of the PALSAR acquisition for Yushu earthquake area and Figure 3.b shows the interferometric phase between January 15, 2010 and July 18, 2010. Figure 3.b presents the severe long-wavelength signals in the far field of the earthquake, which contains the deformation signal, ionospheric and orbital artifacts.

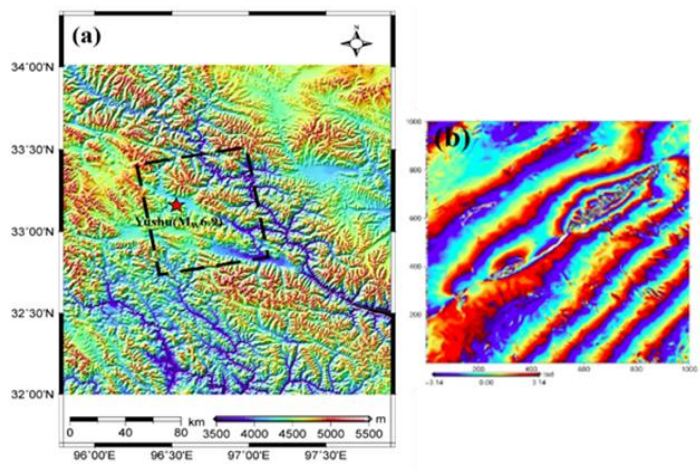

Figure 3. (a) Topographic map of Yushu earthquake area; (b) Interferometric phase.

\subsection{Experimental Result of The Range Split-spectrum Method}

Based on the GAMMA SAR Software Corporation, the narrower bandwidth of 14-MHZ for FBD mode was applied and thus images of FBS mode was down-sampled in our study. Firstly, the range spectrum of the full-band SLCs was split into two sub-band SLCs with the center carrier frequency of \pm 3.5 $\mathrm{MHz}$ and the bandwidth of $6 \mathrm{MHz}$. Since the coherence were not high enough, a precise operation of coregistration were required to recover azimuth offsets induced by the ionosphere. Sub-band interferograms generated by 3 and 15 looks in range and azimuth direction respectively, which were carried out a gold-stein filter with the window of 128 to suppress the noise. After estimating the ionospheric phase delay, the correction of phase unwrapping errors needed carefully manipulate and otherwise the results were in unwanted expectation. In order to remove the wrong information generated by points with low coherence, we applied an outlier detection and mask interpolation with the coherence of 0.8 , and latter a 2-D Gaussian weighted filter required to smooth the ionospheric phase screen.

Figure 4 shows the unwrapped phase of interferometric pair 1 , the unwrapped phase of interferometric pair 2, and the unwrapped phase after compensation of range split-spectrum method for the ionospheric phase delay. Figure 4.d is acquired by subtracting Figure 3.a from Figure 3.b, which can be simply regarded as the phase errors due to the ionospheric disturbance and inaccurate orbit. Figure 4.e is acquired by subtracting Figure 4.a from Figure 4.c. Of course, we can evaluate the performance of the range split-spectrum method by the statistical comparison. The mean and standard deviation values in Figure4.d are respectively $-8.2 \mathrm{rad}$. and $7.5 \mathrm{rad}$, and in Figure 4 .e are respectively $1.2 \mathrm{rad}$ and $2.4 \mathrm{rad}$. We can easily find an encouraging argument provided towards the feasibility for the technique. In order to further compare the phase information before and after correction, the phase values along profile AA' in Figure 4.a-c is extracted, as shown in Figure 4.f. It is noticed that the corrected phase (green line) is closer to the true coseismic deformation phase (blue line) than the original phase (red line).
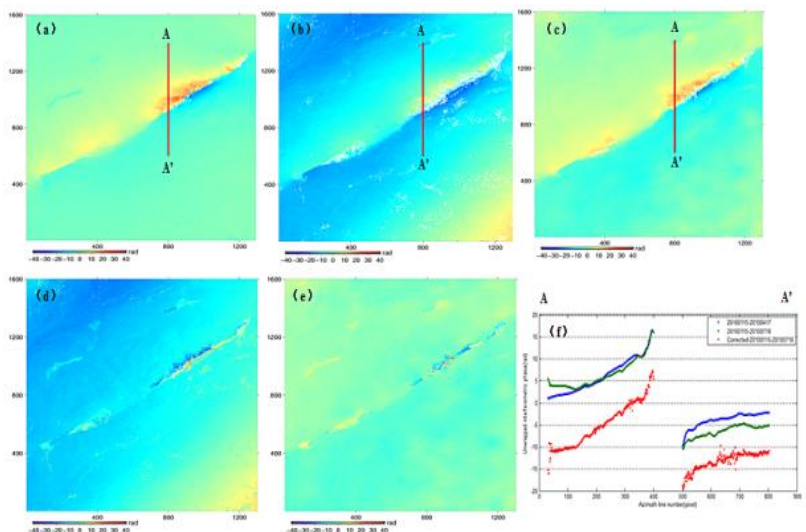

Figure 4. Ionospheric correction for pair 2 in Table 1 by using the range split-spectrum method. (a) Unwrapped phase for interferometric pair 1 in Table 1; (b) unwrapped phase for interferometric pair 2; (c) ionosphere-corrected unwrapped phase for interferometric pair 2 estimated using the range split-spectrum method; (d) phase difference between (a) and (b); (e) phase difference between (a) and (c); (f) the phase values in the profile A-A' of (a)-(c).

\subsection{Experimental Result of The Azimuth Offset Method}

As for the azimuth offset method, the azimuth offset was estimated through Multi-Aperture Interferogram (MAI) technique. Then, the ionospheric contributions on azimuth offsets were separated through directional polynomial fitting. In order to estimate the scaling factor, high coherence points was used. For the integration constant, it was assumed the integration constant as an identical initial value, which is updated by averaging the differences between interferometric phase and initial ionospheric phase delay. After estimation of the azimuth offsets, scaling factor and integration constant, the IPS was estimated and used to correct the ionospheric effect on SAR interferogram (Jung, 2015; Raucoules, 2010).

Figure 5.d is acquired by subtracting Figure 5.a from Figure 5.b which can be simply regarded as the phase errors due to the ionospheric disturbance and inaccurate orbit. Figure 5.e is acquired by subtracting Figure 5.a from Figure 5.c. The mean and standard deviation values in Figure 5.d are respectively $-12.9 \mathrm{rad}$. and $5.3 \mathrm{rad}$. Figure 5.e shows the extracted phase values along profile AA' in a-c. We can also easily find that he corrected phase (green line) is closer to the true coseismic deformation phase (blue line) than the original phase (red line) in the upper part of the earthquake surface rupture. 

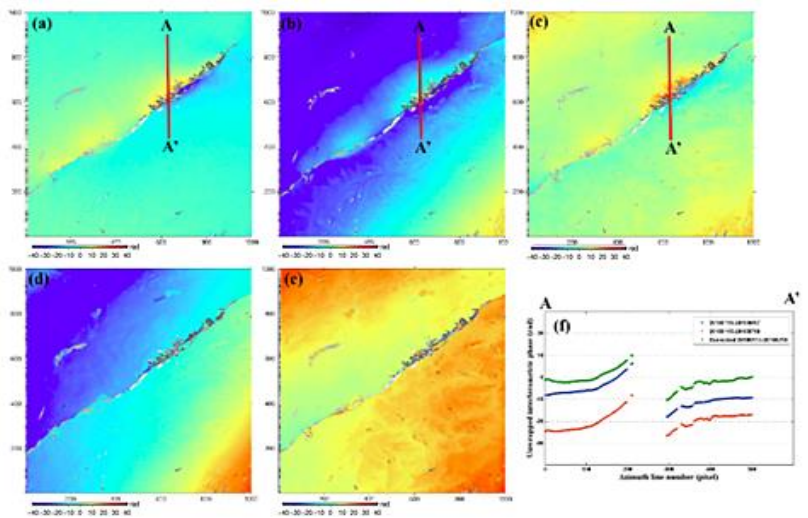

Figure 5. Ionospheric correction by using the azimuth offset method. (a) Unwrapped phase for interferometric pair 1 in Table 1; (b) unwrapped phase for interferometric pair 2; (c) ionosphere-corrected unwrapped phase for interferometric pair 2 estimated using the azimuth offset method; (d) phase difference between (a) and (b); (e) phase difference between (a) and (c); (f) the phase values in the profile A-A' of (a)-(c).

\subsection{Comparison with Results of The Range Split-spectrum and The Azimuth Offset Method}

Comparison with the phases of pair 1 and pair 2 suggest that the two methods both can correct the most of long wavelength ionospheric signals. The phase values along profile $\mathrm{AA}^{\prime}$ in Figure 3 and Figure 4 also demonstrate the efficiency of our correction. In addition, the statistical comparison in table 2 shows that the absolute mean and standard deviation of the corrected phase difference for the range split-spectrum method and the azimuth offset method are above 5 times and 2 times respectively, lower than those before correction of the two techniques.

\begin{tabular}{cccc}
\hline Method & Interferogram & $\begin{array}{c}\text { Mean } \\
\text { (rad.) }\end{array}$ & $\begin{array}{c}\text { Std } \\
(\mathbf{r a d} .)\end{array}$ \\
\hline Range & Original & -8.2 & 7.5 \\
Split-spectrum & Ionosphere-corrected & -1.2 & 2.4 \\
\hline \multirow{2}{*}{ Azimuth Offset } & Original & -12.9 & 5.3 \\
& Ionosphere-corrected & 6.5 & 2.6 \\
\hline
\end{tabular}

Table 2. The mean and standard deviation values of differential interferometric phase before and after ionospheric correction for Yushu coseismic interferogram are shown.

From the result, we can also find that the range split-spectrum method for ionospheric correction is more precise than the other one. We can explain it that the former method is based on the phase in rang and azimuth direction but the latter is only based on the phase in azimuth direction. And also azimuth shifts caused by ionosphere and ground motion are mixed together, which makes it a difficult to use the method on strong surface motion.

However, the corrected images still exit the residual ionospheric signals. For the range split-spectrum method of Yushu earthquake, the ionospheric blurring is so strong and lead to not fine azimuth coregistration, which may cause errors and even help the noise amplification by lowering the coherence of interferograms. Moreover, the phases of sub-band interferograms are not absolute and thus lead to the uncertainty of differential ionospheric delay. When using the azimuth offset to mitigate the ionospheric effect on SAR interferometry, this error may be induced by contribution in the estimated azimuth offset and interferometric phase maps. The residual phase contribution is a limitation of two methods for removing the ionospheric effect and need to be suppressed.

\section{CONCLUSION}

In this paper, we exploit two common methods to correct the ionospheric irregularities. The result indicates that both methods can mitigate the ionospheric effect in SAR interferograms. Absolutely, the experiment that the range split-spectrum method is more effective than the azimuth offset method in the condition of large surface motion.

However, it is also found that the range split-spectrum method is easily contaminated by the noise especially in the condition of poor coherence, and the achievable accuracy of the azimuth offset method is limited by the ambiguous integral constant. Absolutely, many possible works can be extended in various directions, which are shown in the following. On one hand, azimuth offset method can be used to estimate the small scale precise azimuth offsets to recover the coherence of SAR interferogram. On the other hand, the original ionospheric contribution estimated by the range split-spectrum method can help determine the ambiguous integral constant of azimuth offset method, and find an effective technique to separate azimuth offsets caused by ionosphere from the surface motion to improve the accuracy of the azimuth offset method.

\section{ACKNOWLEDGMENTS}

This research was funded by Chang'an university (Xi'an, China) through Natural Science Foundation of China projects (NSFC) (No:41504005,41731066 and 41790445), the Fundamental Research Funds for the Central Universities (No. 310826171005).

\section{REFERENCES}

Bamler, R., \& Eineder, M. (2005). Accuracy of differential shift estimation by correlation and split-bandwidth interferometry for wideband and delta-k sar systems. IEEE Geoscience \& Remote Sensing Letters, 2(2), pp. 151-155.

Brcic, R., Parizzi, A, Eineder, M., Bamler, R., \& Meyer, F. (2010): Estimation and compensation of ionospheric delay for SAR interferometry: Geoscience and Remote Sensing Symposium (Vol.38, pp.2908-2911).

Fattahi, H., Simons, M., \& Agram, P. (2017). Insar time-series estimation of the ionospheric phase delay: an extension of the split range-spectrum technique. IEEE Transactions on Geoscience \& Remote Sensing,PP(99), 1-13.

Gomba, G., Zan, F. D., Gomba, G., \& Zan, F. D. (2017). Bayesian data combination for the estimation of ionospheric effects in sar interferograms. IEEE Transactions on Geoscience \& Remote Sensing, pp. 1-12.

Gomba, G., Parizzi, A., Zan, F. D., Eineder, M., \& Bamler, R. (2016). Toward operational compensation of ionospheric effects in sar interferograms: the split-spectrum method. IEEE Transactions on Geoscience \& Remote Sensing, 54(3), pp.1446-1461. 
Jung, H. S., Lee, D. T., Lu, Z., \& Won, J. S. (2013). Ionospheric correction of sar interferograms by multiple-aperture interferometry. IEEE Transactions on Geoscience \& Remote Sensing, 51(5), pp.3191-3199.

Jung, H. S., \& Lee, W. J. (2015). An improvement of ionospheric phase correction by multiple-aperture interferometry. IEEE Transactions on Geoscience \& Remote Sensing, 53(9), pp. 4952-4960.

Meyer, F. (2010). A review of ionospheric effects in low-frequency SAR - Signals, correction methods, and performance requirements. Geoscience and Remote Sensing Symposium. IEEE, (Vol.38, pp.29-32).

Raucoules, D., \& De Michele, M. (2010). Assessing ionospheric influence on l-band sar data: implications on coseismic displacement measurements of the 2008 sichuan earthquake. IEEE Geoscience \& Remote Sensing Letters, 7(2), pp.286-290.

Rosen, P. A., Hensley, S., \& Chen, C. (2010). Measurement and mitigation of the ionosphere in L-band Interferometric SAR data. Radar Conference (Vol.29, pp.1459-1463). IEEE.

Wright, P. A., Quegan, S., Wheadon, N. S., \& Hall, C. D. (2003). Faraday rotation effects on 1-band spaceborne sar data. Geoscience \& Remote Sensing IEEE Transactions on, 41(12), PP.2735-2744. 